Med Klin Intensivmed 2011 • 106:8-9 DOI 10.1007/s00063-011-0020-y

Online publiziert: 22. September 2011

(c) Springer-Verlag 2011

\author{
U. Janssens ${ }^{1} \cdot$ U. Müller-Werdan ${ }^{2}$ \\ ${ }^{1}$ Klinik für Innere Medizin, St.-Antonius-Hospital, Eschweiler \\ ${ }^{2}$ Universitätsklinik und Poliklinik für Innere Medizin II, Universitätsklinikum \\ Halle (Saale) der Martin-Luther-Universität Halle-Wittenberg, Halle (Saale)
}

\title{
Alt, älter, am ältesten
}

\section{Intensivmedizin vor neuen (alten) Herausforderungen}

Ein hohes Lebensalter bedeutet nicht, dass das Alter von Natur kurz wäre und künstlich verlängert werden müsste, sondern dass die von Natur gesetzten Grenzen erreicht werden. (Lü Buwei, chinesischer Kaufmann, Politiker und Philosoph, etwa 300-235 v. Chr.)

Der alte Patient in der Intensivmedizin ein Randthema oder hochaktuell? Diese Frage werden Sie sich, sehr verehrte Leserinnen und Leser, sicherlich gestellt haben und mit Interesse dieses Heft in die Hand genommen und damit die Antwort auf diese bewusst rhetorisch gestellte Frage gegeben haben.

Natürlich ist dem intensivmedizinisch tätigen Arzt, aber auch den Ärztinnen und Ärzten aller Disziplinen sowohl im stationären wie auch im ambulanten Versorgungsbereich längst bewusst, welchen dramatischen Einfluss die Verschiebung der Alterspyramide mit einer zunehmenden Alterung der Bevölkerung auf die Versorgungsstrukturen im Gesundheitssystem genommen hat, nimmt und nehmen wird. Dabei ist es erstaunlich, mit welcher Gelassenheit sich die Medien, aber auch die Gesundheitspolitiker diesem Thema zuwenden - wenn eine Auseinandersetzung inhaltlicher Art überhaupt stattfindet.

Weltweit nimmt die Zahl der älteren Menschen mit 2\% jährlich wesentlich rascher zu als die Gesamtbevölkerungszahl. Zumindest in den kommenden 25 Jahren wird die Zahl der älteren Menschen voraussichtlich weiterhin stärker ansteigen als die Zahl der Angehörigen ande- rer Altersgruppen. Die Wachstumsrate bei den über 60-Jährigen wird im Zeitraum von 2025 bis 2030 auf jährlich 2,8\% steigen.

Der Alterungsprozess betrifft auch die ältesten Bevölkerungsgruppen: Die weltweit am schnellsten wachsende Altersgruppe ist die der hochbetagten Menschen mit 80 Jahren und darüber. Diese Altersgruppe wächst derzeit jährlich um 3,8\% und macht mehr als ein Zehntel der Gesamtzahl der älteren Menschen aus. Bis zur Mitte dieses Jahrhunderts wird ein Fünftel der älteren Menschen 8o Jahre oder älter sein. Der jährliche Bericht der Europäischen Union zur sozialen Lage in der EU (Internetzitat, s. [2]) weist ebenfalls auf dieses Problem hin. Zwischen 2005 und 2050 wird sich der Anteil der über 80 -Jährigen von 4 auf $11 \%$ verdreifachen.

Die umfangreiche Auswertung der ANZICS (Australia New Zealand Intensive Care Society)-Studiengruppe [1] gibt einen exzellenten Einblick in die aktuelle und zukünftige Altersstruktur von Intensivpatienten und wendet sich schwerpunktmäßig den über 8o-Jährigen zu. Zwischen 2001 und 2005 wurden 120.123 Aufnahmen mit einer Liegedauer von $\geq 1$ Tag analysiert, 57 Intensivstationen nahmen an dieser Untersuchung teil. Von diesen Patienten waren 13\% $\geq 8$ o Jahre alt. Der Anteil dieser Altersgruppe nahm im Beobachtungszeitraum um 5,8\% (3,8$7,3 \%, 95 \%$-Konfidenzintervall) jährlich zu. Diese Steigerungsraten übersetzen sich in eine $72,4 \%$ ige Steigerung des Bedarfs an Intensivbettentagen bis zum Jahr 2015.
Alter per se - so sind sich alle Mediziner und Intensivmediziner einig - stellt keine Indikation zur Ressourcenbegrenzung dar. Dennoch bleiben aus derzeitiger Sicht viele Unsicherheiten, wie künftig mit diesem „Problem“ (nicht nur in der Intensivmedizin) umzugehen ist. Das vorliegende Themenheft soll den Blick für den alten Patienten auf der Intensivstation schärfen. Die gewählten Themen können und sollen natürlich nicht den kompletten Überblick über dieses komplexe Thema abbilden, sind aber als Anstoß zu einer differenzierten Betrachtung in der Therapie kritisch kranker, alter Patienten zu verstehen. Der Beitrag von U. Müller-Werdan et al. ist hier ein Einstieg ins Thema und wendet sich den spezifischen (medizinischen) Problemen betagter Menschen in der Intensivmedizin zu.

Die Pharmakotherapie ist essenzieller Bestandteil der (Intensiv)Medizin, hier verfügen wir (Intensiv)Mediziner oft nicht über eine ausreichende Expertise zu den komplexen medikamentösen Interaktionen und Nebenwirkungen bei schwerstkranken Intensivpatienten, die gerade bei älteren Patienten gravierende und prognosebestimmende Auswirkungen haben können. Daher sind wir $P$. Thürmann und S. Schmiedl besonders dankbar für ihren Beitrag zur Pharmakotherapie alter Patienten. Ergeben sich bei alten Patienten besondere ethische Fragestellungen? Diese und andere Themen werden durch A. Simon in seinem Beitrag aufgearbeitet. Sehr lesenswert sind seine Ausführungen zur immer wieder diskutierten Rationie- 
rung von medizinischen Leistungen bei alten Patienten.

M. Wehler öffnet den Horizont auf das Leben nach der Intensivmedizin bei alten Patienten. Diesen Aspekt vergessen wir immer wieder in unserer täglichen Arbeit. Wir verlegen Patienten auf die Normalstation, in andere Krankenhäuser oder Versorgungseinrichtungen. Wie das Leben der mit höchstem Aufwand behandelten Intensivpatienten nach einer erfolgreichen Intensivtherapie aussieht, entzieht sich regelhaft unserer Kenntnis und muss daher auch in den Fokus gerückt werden.

\section{- Wir wissen viel zu wenig über}

die Besonderheiten in der

Therapie alter Intensivpatienten.

Das muss sich ändern!

Liebe Leserinnen und Leser, wir sind überzeugt, dass Sie dieses Themenheft mit Freude und Interesse lesen werden und viele der benannten Aspekte zu einem späteren Zeitpunkt vertiefen werden. Wir danken den Autoren ausdrücklich für ihre hervorragenden Beiträge und sind sicher, dass diese Informationen Sie auch im klinischen Alltag unterstützen und Sie für die Probleme alter Intensivpatienten sensibilisieren werden. Gleichzeitig wissen wir aber auch, dass noch viel zu tun ist und die zuständigen Fachgesellschaften sich vor dem Hintergrund der aktuellen Entwicklungen noch enger verzahnen und eine aktive Zusammenarbeit aufnehmen und gestalten müssen.

Ihr

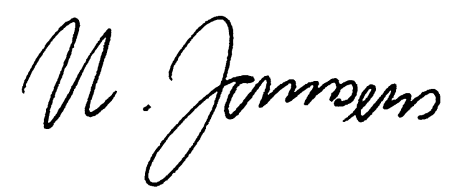

Prof. Dr. Uwe Janssens

Ihre

\section{U. Mïler-Werdan}

Prof. Dr. Ursula Müller-Werdan

\section{Korrespondenzadressen}

\section{Prof. Dr. U. Janssens}

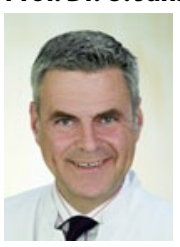

Klinik für Innere Medizin, St.-Antonius-Hospital Dechant-Deckers-Str. 8, 52249 Eschweiler uwe.janssens@ sah-eschweiler.de

\section{Prof. Dr. U. Müller-Werdan}

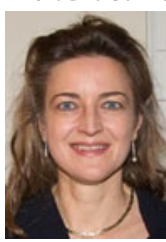

Universitätsklinik und Poliklinik für Innere Medizin II, Universitätsklinikum Halle (Saale) der Martin-LutherUniversität Halle-Wittenberg Ernst-Grube-Str. 40, 06097 Halle (Saale) ursula.mueller-werdan@ medizin.uni-halle.de

\section{Literatur}

1. Bagshaw SM, Webb SA, Delaney A, George C, Pilcher D, Hart GK, Bellomo R (2009) Very old patients admitted to intensive care in Australia and New Zealand: a multi-centre cohort analysis. Crit Care 13:R45

2. Janssens U, Graf J (2010) Alt, älter, am ältesten. Intensiv-News 1:1-5

\section{Herz und Hirn}

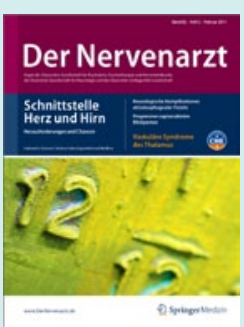

Gleiche Risikofaktoren verursachen vaskuläre Erkrankungen von Herz und Gehirn. Die Faktoren weisen ebenfalls Parallelen hinsichtlich ihrer Pathogenese auf.

Ein Beispiel ist die zerebrale Ischämie, die mit einem hohen Herzinfarktrisiko einhergeht. Umgekehrt haben Patienten mit koronarer Herzkrankheit und Patienten mit Herzinsuffizienz ein erhöhtes Schlaganfallrisiko. Neue Entwicklungen hinsichtlich der Antikoagulation, der antiarrhythmischen Therapie sowie interventioneller Techniken erweitern das therapeutische Spektrum von Kardiologen und Neurologen.

Das Leitthemenheft "Herz und Hirn" (Ausgabe 02/11) der Zeitschrift Der Nervenarzt informiert über Innovationen auf diesem Themengebiet. Es beinhaltet u.a. Beiträge zu folgenden Themen:

•Entwicklung von Mortalität und Morbidität vaskulärer Erkrankungen

-Medikamentenadhärenz bei chronischen Erkrankungen

-Epidemiologie, Klinik und Management des Vorhofflimmerns

-Detektion von Vorhofflimmern beim Schlaganfall

-Techniken zum Verschluss des linken Herzohres

-Neue orale Antikoagulanzien beim Vorhofflimmern

-Kognitive Störungen nach kardiochirurgischen Eingriffen

Bestellen Sie diese Ausgabe zum Preis von EUR 34 zzgl. Versandkosten bei:

Springer Customer Service Center Kundenservice Zeitschriften

Haberstr. 7

69126 Heidelberg

Tel.: +49 6221-345-4303

Fax: +49 6221-345-4229

E-Mail: leserservice@springer.com

PS: Vieles mehr rund um Ihr Fachgebiet finden Sie auf www.springermedizin.de 